\title{
Microsurgical Outcomes of Secondary Epilepsy from Hippocampal Lesions: A Report of 56 Cases and Literature Review
}

\author{
Jun WANG, De-Guang XING, Er-Meng MA, Bo QIU, Shao-Wu OU, Yun-Jie WANG \\ The First Hospital of China Medical University, Department of Neurosurgery, Shenyang, China
}

\section{ABSTRACT}

AIM: To explore the treatment efficacy of microsurgery for secondary epilepsy from hippocampal lesions.

MATERIAL and METHODS: The clinical data, pathological findings, surgical methods and surgical outcomes of 56 patients with secondary epilepsy from hippocampal lesions were retrospectively analyzed.

RESULTS: Postoperative pathological examinations confirmed that 27 patients had gliomas, 17 patients had vascular malformations and 12 patients had hippocampal sclerosis. Twenty-nine patients underwent selective resection of the lesioned tissue and the surrounding infiltrated tissue, and 26 patients underwent a more generous removal of the anterior temporal lobe, lesioned tissue, infiltrated tissue and medial structures of the temporal lobe. Fifty patients were followed up with an average follow-up duration of 25.5 months. At postoperative one year, the remission rate of epilepsy that achieved Engel grade I was $80.8 \%(21 / 26)$ and $83.3 \%(20 / 24)$ for the selective resection and more generous resection, respectively, indicating that the difference between the two methods was insignificant.

CONCLUSION: Microsurgery is the first choice for the treatment of secondary epilepsy from hippocampal lesions. Various operative routes and methods can be selected based on the lesion natures. Long-term favorable outcome of seizure control following microsurgery can be achieved in most of the patients.

KEYWORDS: Epilepsy, Hippocampus, Microsurgery

\section{INTRODUCTION}

Mesial temporal lobe epilepsy (MTLE) is the most common type of temporal lobe epilepsy in the clinic, and it tends to develop into medically intractable epilepsy. Hippocampal lesions are the most common cause of MTLE. Currently, the treatment strategy of this epilepsy type remains clinically controversial (16). In this paper, we retrospectively analyzed the clinical data and the outcomes of the microsurgical treatment of 56 patients who had secondary epilepsy from hippocampal lesions and were treated during the period from January 2005 to January 2014. The purpose of this study was to explore the significance of microsurgery and its various operative methods in the treatment of secondary epilepsy from hippocampal lesions.

\section{MATERIAL and METHODS}

\section{Selection of Patients}

There were 56 patients enrolled in this study including 31 males and 25 females. These patients were aged 16 to 74 years (mean age, 35.1 \pm 13.5 years) and had a history of epilepsy from 2 months to 10 years (mean length, 17.2 \pm 13.1 months). The inclusion criteria for patients diagnosed as having hippocampal sclerosis was that systematic anti- 
epilepsy treatment had been performed at least for two years before surgery and therefore the history of epilepsy for these patients was ranged from 2.5 to 10 years, (mean length, $40.2 \pm 15.1$ months). Meanwhile, for patients with tumors or vascular malformations, the inclusion criteria was that magnetic resonance imaging (MRI) showed the typical lesions and the clinical manifestation of epilepsy was definite.

\section{Clinical Manifestations}

Of the 56 enrolled patients, 27 had complex partial epilepsy, 6 had simple partial epilepsy, 11 had absence seizures and 12 had secondarily generalized tonic-clonic seizures (including the patients with mixed symptoms, Table I). Before the surgery, the diseases of 18 patients were accompanied by headache, three by nausea and vomiting; three by visual field defects (homonymous hemianopia), two by language impairments, and one by muscle strength declination of the contralateral limbs, and one patient had a history of subarachnoid hemorrhaging.

\section{Imaging Findings}

Prior to surgery, all patients underwent head MRl; seventeen patients also underwent magnetic resonance spectroscopy (MRS), and four underwent cerebral digital subtraction angiography (DSA). Of the 56 patients, twenty-five patients had lesions on the left side, and 31 had lesions on their right side. Except for the patients with primary hippocampal sclerosis, the imaging examination demonstrated that seven patients had lesions localized at the head of the hippocampus (Figures 1A-P; 2A-P; 3A-T), four had lesions localized at the body of the hippocampus, three had lesions in both the head and body of the hippocampus, and four had lesions in the head, body and tail of the hippocampus. Furthermore, the white matter of the hippocampus and temporal lobe were involved in the disease in 21 patients, both the white matter and cortex in the hippocampus and temporal lobe were involved in seven patients, both the white matter and cortex in the hippocampus and temporal lobe, as well as the insular

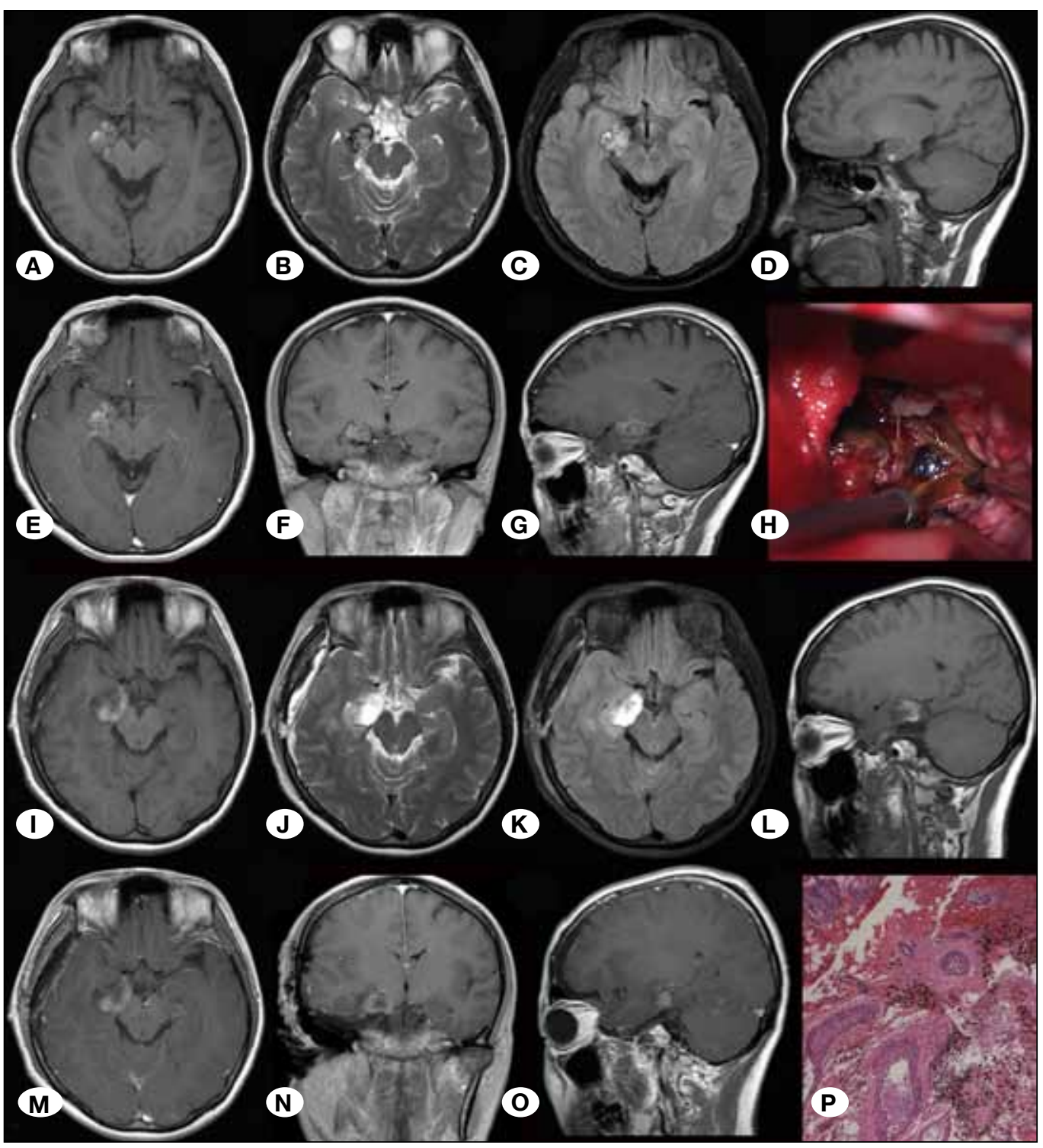

Figure 1: Pre- and postoperative images of cavernous hemangioma in the head of hippocampus. A-G: Pre-operative MRI images showing the lesion located in the head of hippocampus with mixed T1 and T2 signals, as well as no clear enhancement.

H) Intraoperative image shows a vascular lesion (spongy).

I-O) Post-operative MRI images showing the lesion was resected completely and the surrounding structures were preserved. P) Pathology confirming the diagnosis of cavernous hemangioma, H\&E staining, $\times 40$. 


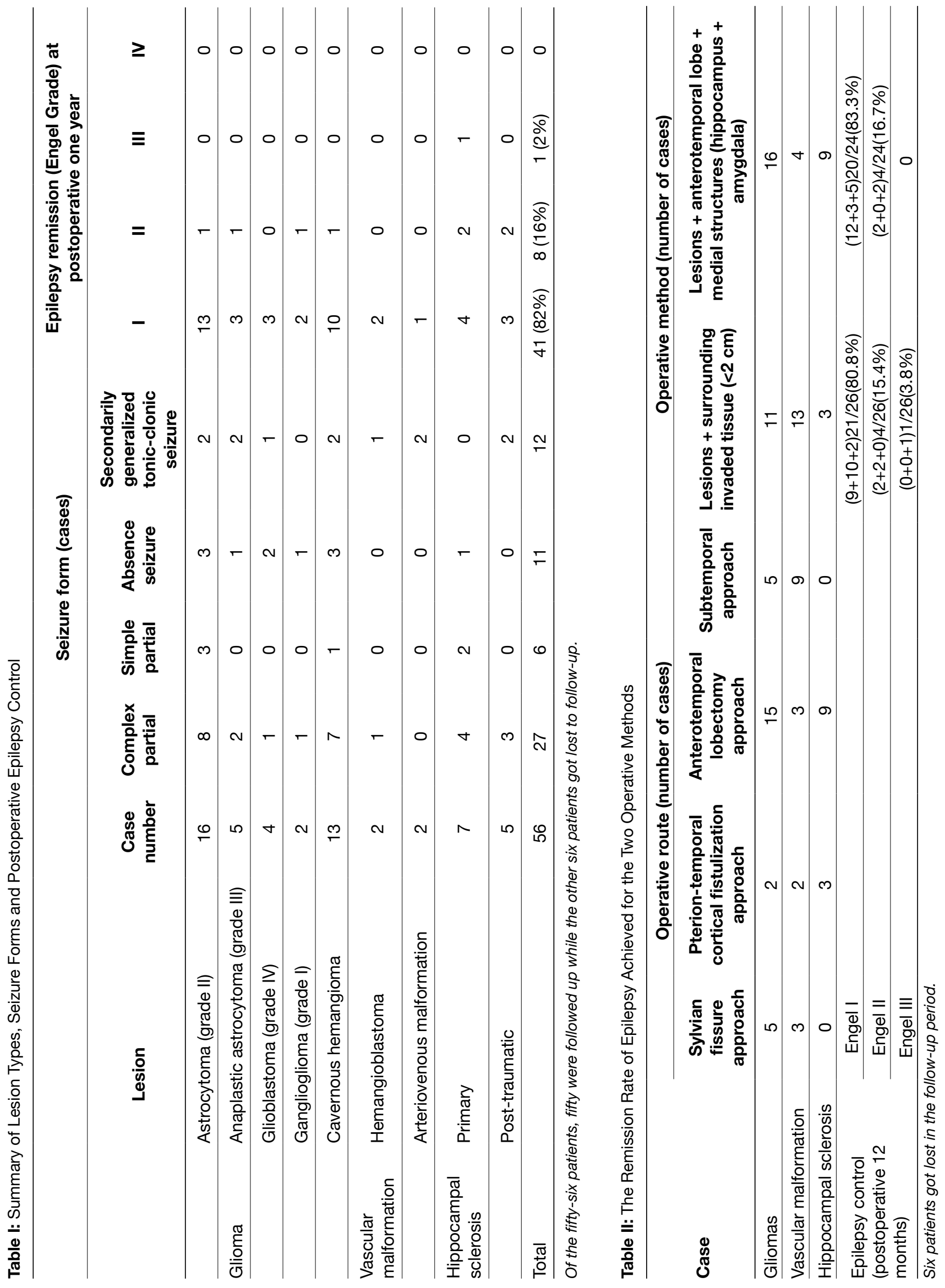


lobe, were involved in three patients (Figure 4A-L), and a wide range of areas, including the hippocampus, temporal lobe, insular lobe and frontal lobe, were involved in the disease of two patients. A preoperative electroencephalography (EEG) was performed on all patients, and among them, twelve patients underwent long-term video-EEG monitoring. Typical epileptiform discharges were recorded in 42 patients.

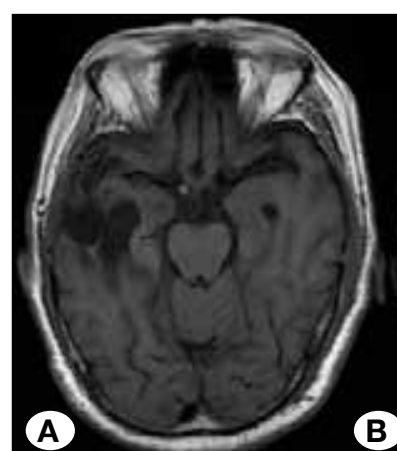

(B)
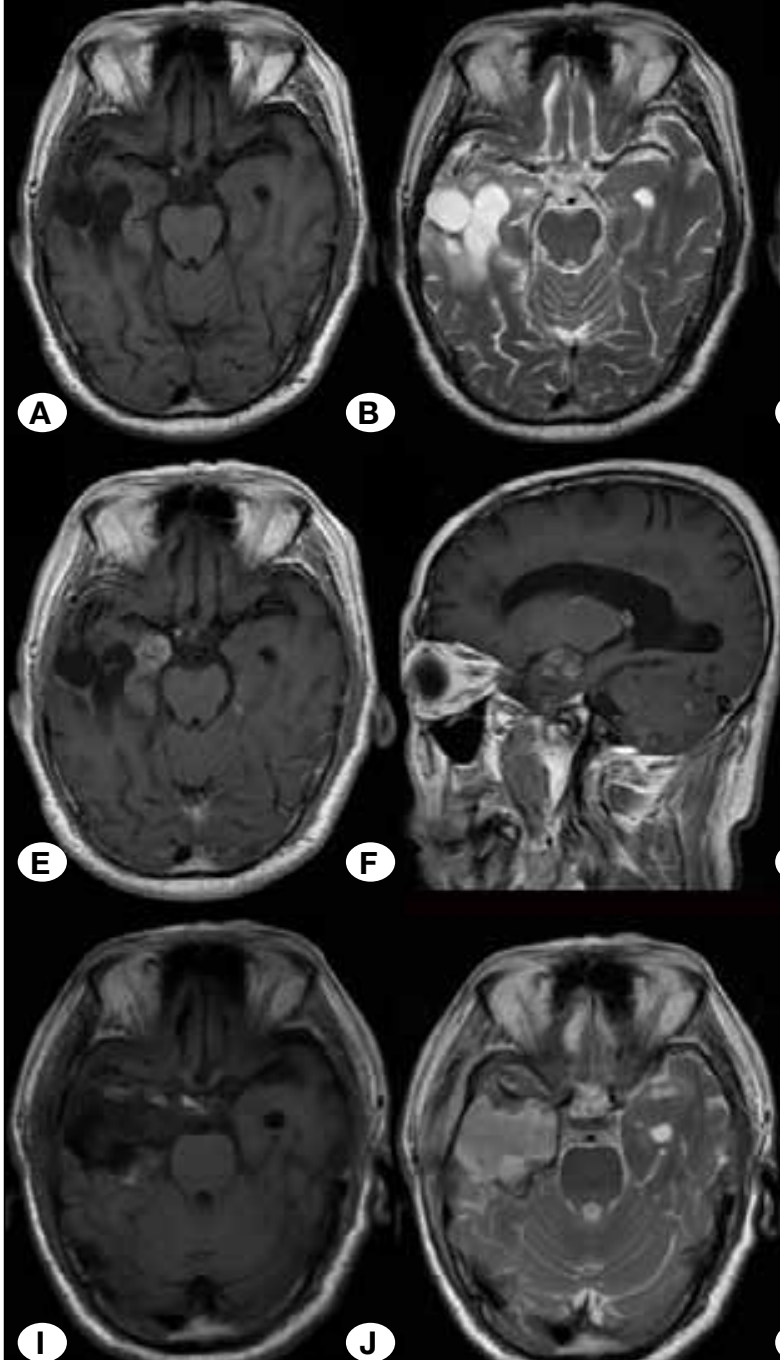

J)

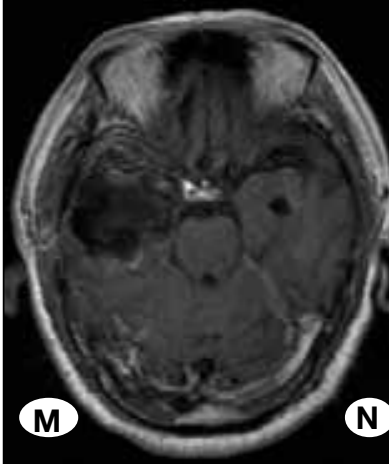

(N)
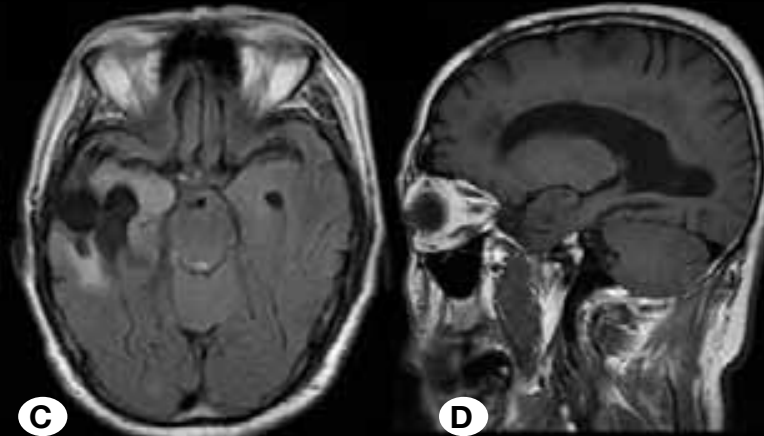

G

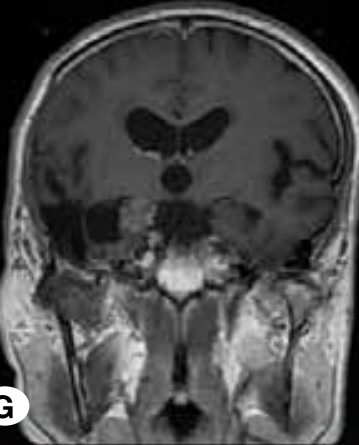

(H)

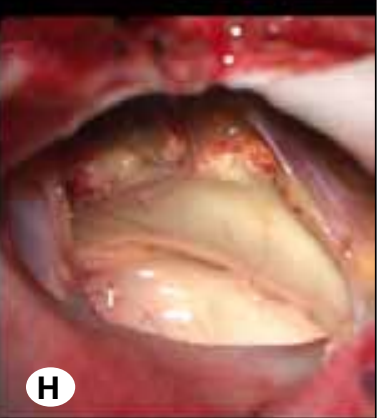

K
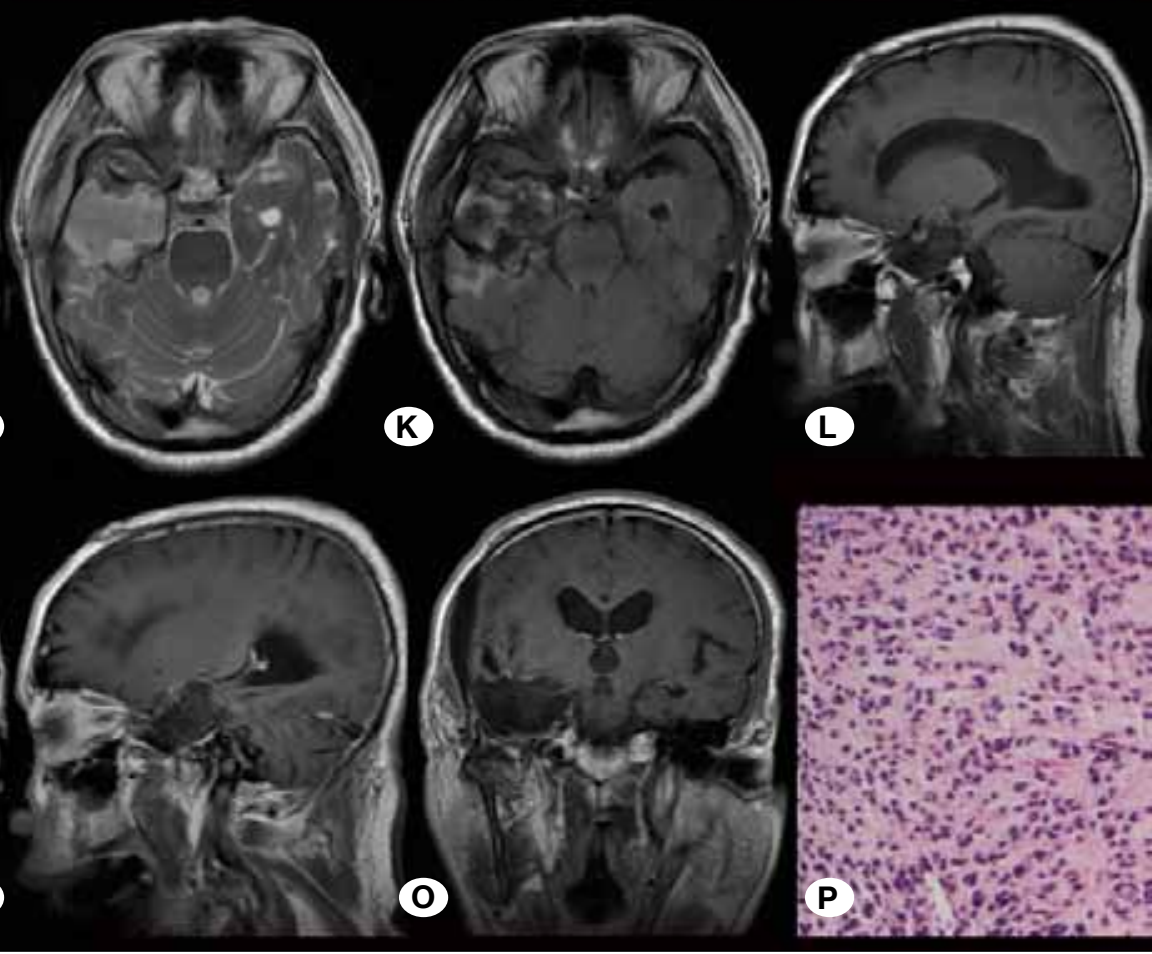

Figure 2: Pre- and post-operative images of Grade III anaplastic astrocytoma in the head of hippocampus. A-G) Pre-operative MRI images showing the lesion located in the head of hippocampus with long T1 and T2 signals, as well as significant enhancement. H) Intraoperative image shows a grey-red lesion. I-O) Postoperative MRI images shows the lesion and the anterior temporal lobe was resected completely. P) Pathology confirming the diagnosis of anaplastic astrocytoma, H\&E staining, $\times 40$. 


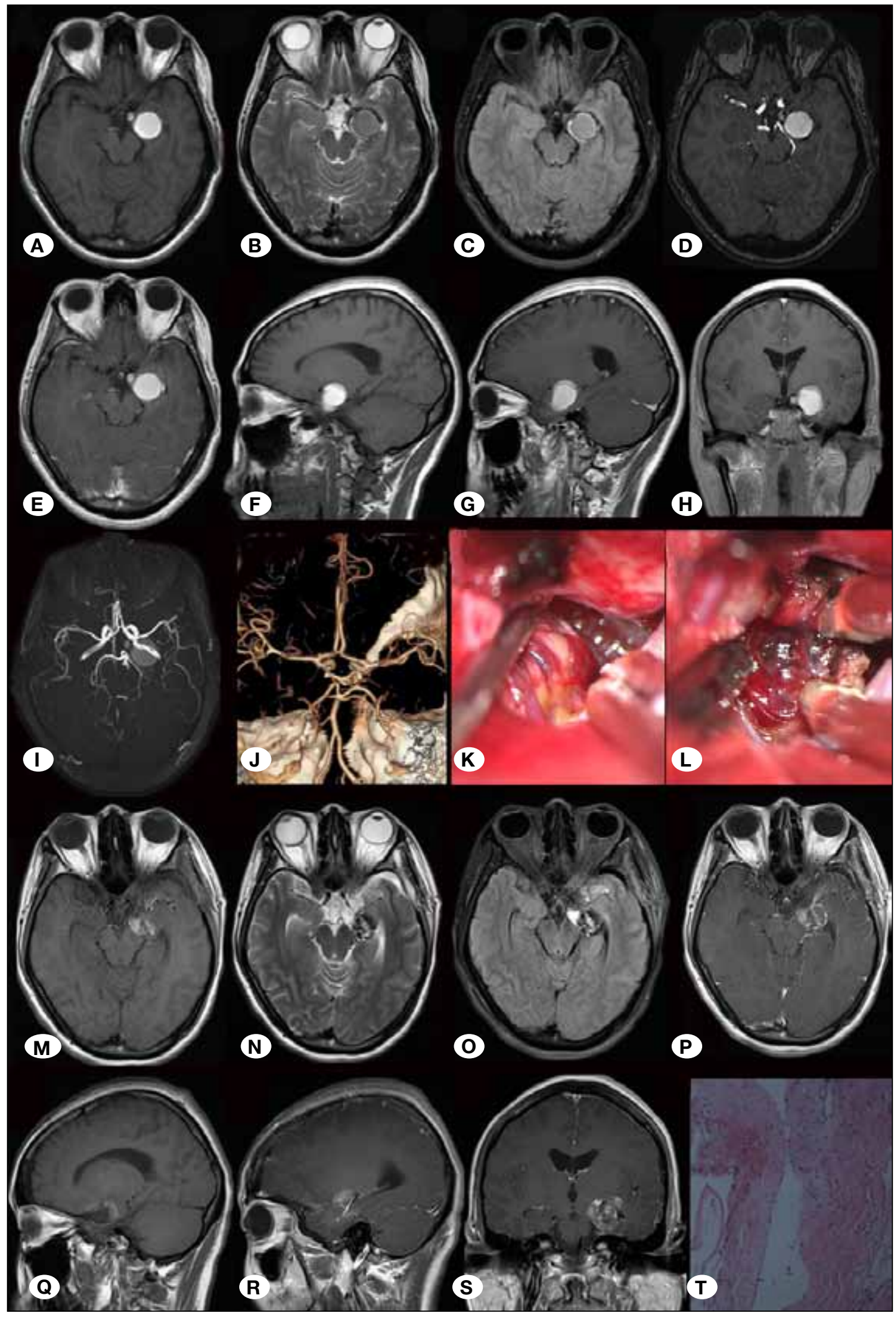

Figure 3: Pre- and post-operative images of hemangioblastoma in the head of hippocampus. A-G) Pre-operative MRI, MRA and 3D-CTA images showing a well-circumscribed lesion located in the head of hippocampus with short T1 and equal T2 signals. K,L) Intraoperative image shows a vascular lesion with thick-walled structures. M-S) Postoperative MRI images shows the lesion was resected completely with a small amount of residual blood. T) Pathology confirming the diagnosis of hemangioblastoma, H\&E staining, $\times 40$. 


\section{Operative Methods}

All 56 patients underwent microsurgery by a single neurosurgery team. The pterional-sylvian fissure approach was adopted in eight patients, and the pterional-temporal cortical fistulization approach was adopted in seven patients. The approach for temporal lobe resection through the pterion was adopted in 27 patients, and the subtemporal approach was adopted in 14 patients. Two operative methods were applied: twenty-seven patients underwent a simple resection of lesions and the surrounding invaded tissue, and 29 patients underwent the more generous resection of the anterior temporal lobe, lesions, invaded tissue and the medial structures of the temporal lobe (Table II, Figures 2A-P; 3A-T). Intraoperative cortical or deep EEG was recorded pre- and post lesion resection.

\section{RESULTS}

\section{Postoperative Pathological Findings and Surgical Resection Range}

Twenty-seven patients were diagnosed with gliomas, including 16 with astrocytoma (grade II), five with anaplastic astrocytoma (grade III; Figure 1A-P), four with glioblastoma (grade IV; Figure 3A-T) and two with ganglioglioma (grade I). A complete surgical removal was conducted in 20 patients, and an incomplete removal was conducted in 7 patients. All patients in this sub-group were recommended for radiotherapy and chemotherapy after surgery. Of the 27 patients, nine decided to take chemotherapy while five chose radiotherapy and ten received chemotherapy and radiotherapy simultaneously. The

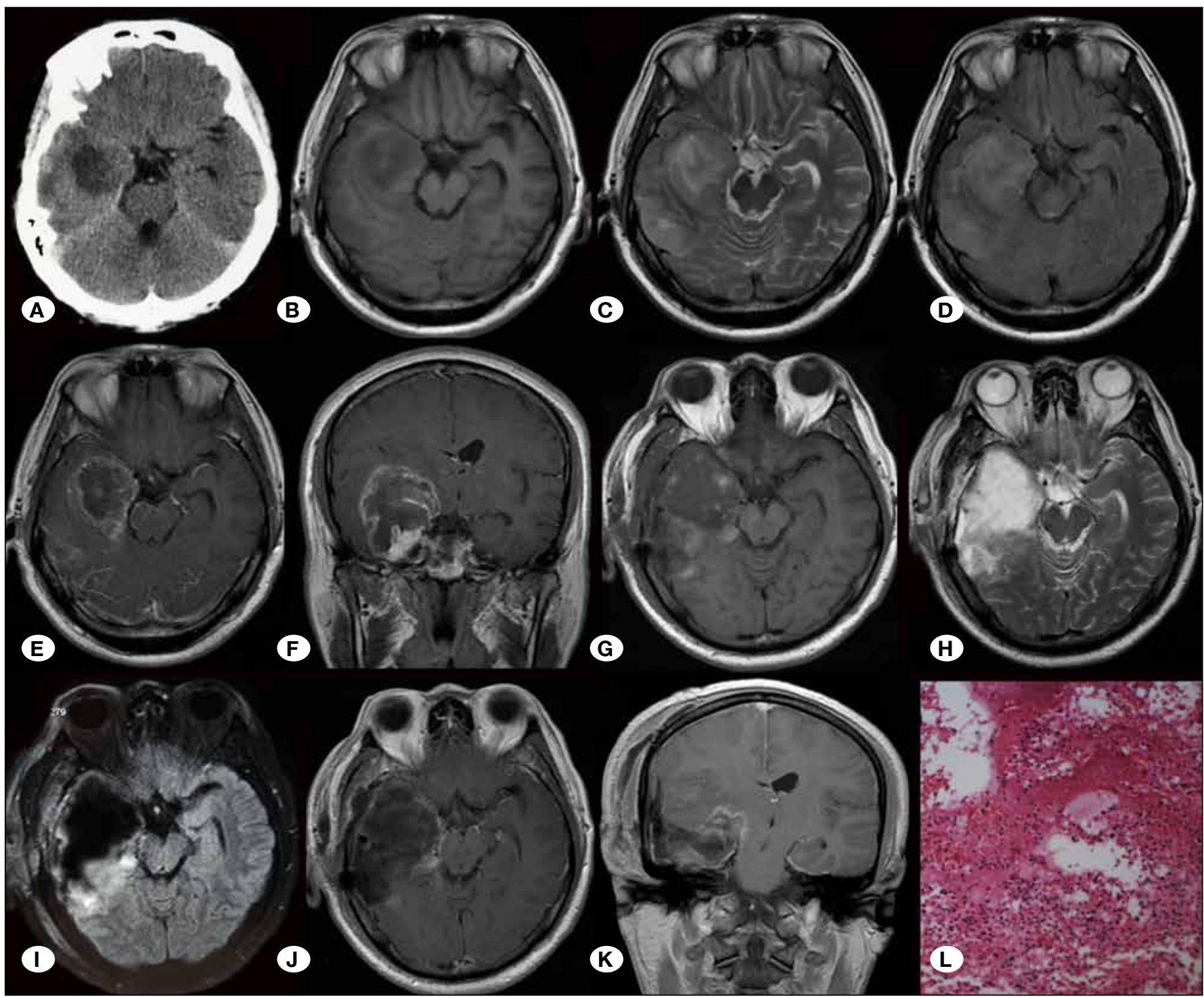

Figure 4: Pre- and post-operative images of glioblastoma in the hippocampus area, with invasions of insular and temporal lobe. A-F) Pre-operative CT and MRI images showing a lesion in the hippocampus area with long T1, equal T2 signals with significant enhancement. The lesion also invades the insular and temporal lobes. E-K) Postoperative MRI images shows the lesion and the anterior temporal lobe was resected completely. L) Pathology confirming the diagnosis of glioblastoma, H\&E staining, $\times 40$. 
other three patients decided to abandon chemotherapy or radiotherapy. Of the 9 patients with gliomas in grade III or IV, one patient suffered from seizure recurrence at one year follow-up and two patients died from tumor recurrence within one and a half years. Five patients were followed up for more than two years, in which four patients suffered from tumor recurrence but not seizures and four of them underwent the second operation. Seventeen patients were diagnosed with vascular malformation, including 13 with cavernous hemangioma (Figure 2A-P), two with hemangioblastoma (Figure 4A-L) and two with arteriovenous malformation. All 17 patients underwent complete surgical removal. Twelve patients were diagnosed with hippocampal sclerosis, including seven with primary hippocampal sclerosis (Figure $5 A-G$ ) and five with post-trauma secondary hippocampal sclerosis. The selective resection of the hippocampus and amygdala was performed in three patients, and the standard resection of the anterior temporal lobe and the medial structures (hippocampus and amygdala) was performed in nine patients (Figure 5A-G).

\section{Postoperative Epilepsy Remission}

Fifty patients were followed up and the follow-up duration was more than one year, with an average follow-up duration of 25.5 months. Twenty nine patients were followed up for more than two years. The remission rate at postoperative one year was as follows: 41 (82\%) patients were classified as Engel grade I, $8(16 \%)$ as Engel grade II and one (2\%) as Engel grade III. The remission rate of epilepsy that achieved Engel grade I was $80.8 \%(21 / 26)$ and $83.3 \%(20 / 24)$ for the selective resection and more generous resection, respectively, indicating that the difference between the two methods was insignificant. Of the 29 patients with a follow-up duration of more than two years, the remission rate classified as Engel grade I at postoperative two years was $82.8 \%(24 / 29)$.

\section{Postoperative Complications}

Postoperative cranial inflammation occurred in six patients, which was controlled by anti-inflammatory treatment and CSF drainage through lumbar puncture or from the lumbar cistern. One patient had postoperative bleeding at the surgical site and eventually died of secondary hydrocephalus and inflammation after surgical hematoma evacuation. Four patients experienced postoperative transient oculomotor nerve paralysis, which improved in three patients at their discharge from the hospital and was relieved one month after the surgery in one patient. One patient exhibited a paralysis of the contralateral upper limb immediately after the surgery and had muscle strength at grade II, which was completely restored three months after the surgery. Four patients had a newly developed transient aphasia, which was eliminated at the time of their hospital discharge. Three patients experienced newly developed memory impairment, which was significantly improved in two patients three months after the surgery but was not improved satisfactorily in another patient even at the follow-up six months after surgery. In addition to the three patients who had a hemianopsia preoperatively (18 patients underwent a visual field examination before surgery), three patients (13 patients underwent visual field examination after surgery) had newly-developed quadranopia after the surgery.

\section{DISCUSSION}

\section{Clinical Manifestations and Classifications of Hippocampal Lesions}

The common clinical types of hippocampal lesions include tumorous lesions (16), vascular lesions $(5,12)$, congenital lesions $(6,8)$, traumatic lesions and others. In different treatment centers, the statistical data regarding the distribution of lesion types differ. In this study, all study subjects were collected from a treatment center focusing on intracranial (brain) tumors; thus, the tumorous lesions accounted for $50 \%$ of hippocampal lesions. Due to admission bias, the order of clinical types of hippocampal lesions would differ in terms of incidence in other treatment centers that focus on epilepsy or cerebrovascular diseases.

The clinical manifestations of hippocampal lesions are very complex. Only patients accompanied by epilepsy were enrolled in this study. For patients diagnosed as having hippocampal sclerosis, systematic anti-epilepsy treatment has been performed at least for two years, which was indicated to be drug-resistant, before neurosurgery. There are various clinical forms of epilepsy. Previous studies have reported that the majority of secondary epilepsy from hippocampal lesions belongs to MTLE, which mainly consists of partial seizures and rarely generalized seizures; furthermore, the predominant partial seizures are dominated by complex partial seizures $(8,12,16)$. In this study, 27 patients had complex partial seizures, accounting for $50 \%$ of the total number of patients, followed by absence seizures and secondarily generalized tonic-clonic seizures; simple partial seizures were relatively rare, consistent with previous reports.

The definitive location-based classification of hippocampal lesions remains controversial. Some scholars classify the hippocampal tumorous lesions into four types, namely, type A, B, C and D (9). Type A refers to lesions localized at the head of the hippocampus, parahippocampal gyrus, uncinate gyrus and amygdala, as well as their anteromedial structures, with the lateral border on the collateral fissure and the upper border on the line connecting the circular sulcus and choroidal fissure. Type $B$ is defined as lesions located at the posterolateral sites of the type A area described as above, including the intermediolateral sulcus collateralis at the medial temporal pole and fusiform gyrus with the lateral border not yet reaching the inferior temporal gyrus. Type $C$ is defined as lesions involving both type $A$ and type $B$ areas with the lateral border not passing the occipitotemporal sulcus. Type $D$ refers to lesions involving the temporal cortex (or subcortex), temporal stem, insular lobe and capsula interna, etc. According to this classification system, in this study, seven patients had type A lesions, four had type $B$ lesions, seven had type $C$ lesions, and 38 had type $\mathrm{D}$ lesions. We believe this classification system has certain guiding significance for the selection of operative routes despite the fact that it is insignificant in determining the lesion nature.

\section{Selection of the Operative Routes for Treating Hippocampal Lesions}

Currently, the operative routes applied in the treatment of 


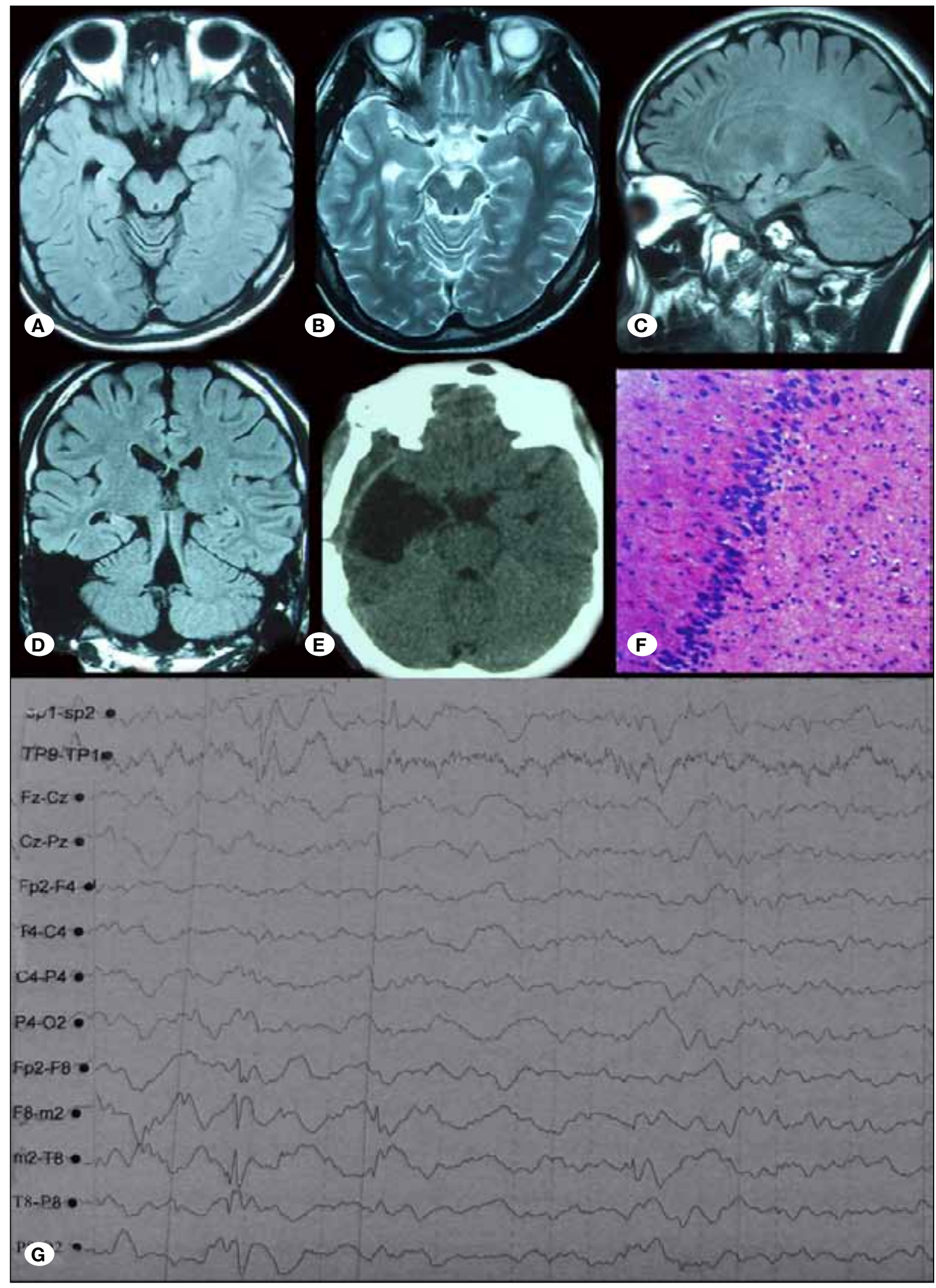

Figure 5: Pre- and postoperative images of primary hippocampal sclerosis. A-D) Preoperative MRI images showing the volume of right hippocampus was smaller than the left one, with an increased signal in the FLAIR scan. E) Standard anterior temporal lobectomy and amygdalohippocampectomy was undertaken. F) Pathology confirming the diagnosis of hippocampal sclerosis, H\&E staining, $\times 40$. G) Preoperative scalp EEG showing the epileptic discharge on the right temporal area. 
hippocampal lesions mainly include the following types: the pterional-sylvian fissure approach (15); the pterionsuperior temporal sulcus (gyrus temporalis medius) cortical fistulization approach (7); the subtemporal approach (4); the pterion-anterotemporal lobectomy approach; and the pterional-sylvian fissure-temporal stem approach. In this study, four approaches including the sylvian fissure approach, the superior temporal sulcus (gyrus temporalis medius) cortical fistulization approach, the subtemporal approach and the pterion-anterotemporal lobectomy approach served as the main operative routes. The pterional-sylvian fissure approach was applied in eight patients, the pterion-temporal cortical fistulization approach in seven patients, the pterionanterotemporal lobectomy approach in 27 patients and the subtemporal approach in 14 patients. We believe that all of the above approaches are suitable for lesions localized in the head of hippocampus (namely, type A lesions) and can be selected based on the degree of familiarity and operating habit of the surgeons. With regard to the lesions localized in the body of the hippocampus or involving the entire hippocampus (type B or C lesions), these four approaches are applicable; moreover, we believe the subtemporal approach can be the first selection due to the more convenient operation for the exposure and resection of lesions. With regard to the lesions involving a broader area, such as type C or D lesions, the pterion-anterotemporal lobectomy approach should be more convenient and appropriate.

Each operative route has advantages and disadvantages. For example, the pterional-sylvian fissure approach, which has been widely used in the clinic, requires a high level of microsurgical skills in the treatment of hippocampal lesions. In particular, during the operation, the middle cerebral artery and its branches to the insular lobe and temporal lobe, as well as oculomotor nerves, must be carefully protected (15). The advantage of this approach is that the operation-related injuries on the lateral temporal cortex, optic radiation and its fiber connections are relatively less, leading to the maximal functional preservation of the lateral temporal cortex. The pterional-temporal cortical fistulization approach was used in the surgical resection of medial structures, such as the hippocampus and amygdala, early and widely in the clinic. This approach has merits, such as an easy operation and easy positioning of the hippocampus after ventricle exposure (7), but also has shortcomings, such as serious damage of the temporal cortex. The subtemporal approach can expose hippocampal lesions from the subtemporal incision without the involvement of the temporal cortex, the middle cerebral artery and optic radiation; however, the pulling of the temporal lobe when needed might cause damage to the temporal drainage veins, such as the Labbé vein, which can result in complications, including temporal edema and hemorrhage (4).

Therefore, we believe that the operative approaches should not be adopted blindly; instead, the selection should comprehensively take into account the location and nature of the lesions, as well as surgeon experience.

\section{The Effect of Different Operative Approaches and Pathologic Types on the Epilepsy Remission Rate}

The selection of operative approaches for hippocampal lesions in treating epilepsy has been controversial. Previous studies have found that for patients with secondary temporal epilepsy from only hippocampal sclerosis, there was no significant difference in the clinical remission rate of epilepsy between the patients receiving the selective resection of the hippocampus and amygdala and the patients receiving the standard resection of anterotemporal medial structures $(1,3,10,11,13,14)$. Two surgical methods were adopted in this study: 27 patients underwent the simple resection of lesions and the surrounding invaded tissue, and 29 patients underwent standard resection of the anterior temporal lobe, lesions and their surrounding invaded tissue as well as the medial structures of the temporal lobe. No significant difference in the epilepsy remission rate was observed during the postoperative one year follow-up between the two methods (Engel I: $80.8 \%(21 / 26)$ vs. $83.3 \%(20 / 24))$, which might be related to the fact that all enrolled patients had secondary epilepsy from hippocampus lesions, as confirmed by hippocampal imaging. In order to explore whether there was a statistical difference in the epilepsy remission rate between various pathologic types, we analyzed the surgical outcomes of epilepsy with different pathologic lesions. As indicated in Table II, the remission rate of epilepsy that achieved Engel grade $I$ at postoperative one year was $21 / 25(84 \%), 13 / 15$ $(86.6 \%)$ and $7 / 10(70 \%)$ for the group of gliomas, vascular malformations and hippocampal sclerosis, respectively. It seems that the epilepsy remission rate after surgery was similar in the group of glioma and vascular malformations and was much better than that in the hippocampus sclerosis group; however, since the sample size was small in varying pathologies, such as vascular malformations or hippocampal sclerosis, the outcomes in relation to different pathologies was just analyzed preliminarily. Therefore, these results still need to be determined by analyzing more surgical cases with different pathologic lesions. Most previous studies have stated that the epileptic foci of MTLE caused by the tumorous, vascular and traumatic hippocampal lesions are mostly located within the lesioned tissue or the adjacent invaded brain tissue $(<2$ $\mathrm{cm}$ ); as a result, the epileptic foci could be removed during the simple resection of lesions and the surrounding invaded tissue. Therefore, the effectiveness of epilepsy control is not necessarily related to the anterior temporal lobectomy. However, for the patients with secondary temporal epilepsy from congenital lesions, such as malformations of temporal cortical development complicated with hippocampal sclerosis, or the patients whose lesions are undetectable by MRI but with secondary temporal epilepsy diagnosed based on seizure semiology and through intracranial electrode implantation (2), the epilepsy foci might not be localized in the medial temporal lobe. Therefore, most scholars recommend the standard resection of the anterior temporal lobe and medial structures.

In summary, the surgical treatment is recommended as the first choice for the patients with secondary temporal epilepsy from hippocampal lesions. The selection of operative routes and methods should be based on the lesion nature and 
location. Epilepsy can be improved after surgical treatment in most patients.

\section{- ACKNOWLEDGMENTS}

This study has been supported by the National Natural Science Foundation of China (No. 31100770) and the Liaoning Provincial Natural Science Foundation of China (No. 2014021097). We thank all the other staff of the neurosurgery department of the First Hospital of China Medical University for their technical help.

\section{- REFERENCES}

1. Bandt SK, Werner N, Dines J, Rashid S, Eisenman LN, Hogan $\mathrm{RE}$, Leuthardt EC, Dowling J: Trans-middle temporal gyrus selective amygdalohippocampectomy for medically intractable mesial temporal lobe epilepsy in adults: Seizure response rates, complications, and neuropsychological outcomes. Epilepsy Behav 28:17-21, 2013

2. Cohen-Gadol AA, Spencer DD: Use of an anteromedial subdural strip electrode in the evaluation of medial temporal lobe epilepsy. Technical note. J Neurosurg 99:921-923, 2003

3. Elsharkawy AE, Pannek H, Woermann FG, Gyimesi C, Hartmann S, Aengenendt J, Ogutu T, Hoppe M, Schulz R, Pietilä TA, Ebner A: Apical temporal lobe resection; "tailored" hippocampus-sparing resection based on presurgical evaluation data. Acta Neurochir (Wien) 153:231-238, 2011

4. Hori T, Yamane F, Ochiai T, Kondo S, Shimizu S, Ishii K, Miyata $\mathrm{H}$ : Selective subtemporal amygdalohippo- campectomy for refractory temporal lobe epilepsy: Operative and neuropsychological outcomes. J Neurosurg 106:134-141, 2007

5. Lopez-Ojeda P, Labib M, Burneo J, Lownie SP: Temporal lobe arteriovenous malformations: Surgical outcomes with a focus on visual field defects and epilepsy. Neurosurgery 73:854-862, 2013

6. Miyata H, Hori T, Vinters HV: Surgical pathology of epilepsyassociated non-neoplastic cerebral lesions: A brief introduction with special reference to hippocampal sclerosis and focal cortical dysplasia. Neuropathology 33:442-458, 2013
7. Niemeyer P: The transventricular amygdala-hippocampectomy in temporal lobe Epilepsy. Springfield, IL: Charles C. Thomas, 1958

8. Ramey WL, Martirosyan NL, Lieu CM, Hasham HA, Lemole GM $\mathrm{Jr}$, Weinand ME: Current management and surgical outcomes of medically intractable epilepsy. Clin Neurol Neurosurg 115:24112418, 2013

9. Schramm J, Aliashkevich AF: Temporal mediobasal tumors: A proposal for classification according to surgical anatomy. Acta Neurochir (Wien) 150:857-864, 2008

10. Spencer D, Burchiel K: Selective amygdalohippocampectomy. Epilepsy Res Treat 2012:382095, 2012

11. Tanriverdi T, Olivier A, Poulin N, Andermann F, Dubeau F: Longterm seizure outcome after mesial temporal lobe epilepsy surgery: Corticalamygdalo- hippocampectomy versus selective amygdalohippocampectomy. J Neurosurg 108:517-524, 2008

12. von der Brelie C, Malter MP, Niehusmann P, Elger CE, von Lehe $\mathrm{M}$, Schramm J: Surgical management and long-term seizure outcome after epilepsy surgery for different types of epilepsy associated with cerebral cavernous malformations. Epilepsia 54:1699-1706, 2013

13. Wendling AS, Hirsch E, Wisniewski I, Davanture C, Ofer I, Zentner J, Bilic S, Scholly J, Staack AM, Valenti MP, Schulze-Bonhage A, Kehrli P, Steinhoff BJ: Selective amygdalohippocampectomy versus standard temporal lobectomy in patients with mesial temporal lobe epilepsy and unilateral hippocampal sclerosis. Epilepsy Res 104:94-104, 2013

14. Yaşargil MG, Krayenbühl N, Roth P, Hsu SP, Yaşargil DC: The selective amygdalohippocampectomy for intractable temporal limbic seizures. J Neurosurg 112:168-185, 2010

15. Yasargil MG, Teddy PJ, Roth P: Selective amygdalohippocampectomy. Operative anatomy and surgical technique. Adv Tech Stand Neurosurg 12:93-123, 1985

16. Zaatreh MM, Firlik KS, Spencer DD, Spencer SS: Temporal lobe tumoral epilepsy: Characteristics and predictors of surgical outcome. Neurology 61:636-641, 2003 